\title{
A Novel Colored Time Petri Net Model for Policy Management and Context Awareness Procedure in MANET
}

\author{
Jing Wang, Huyin Zhang*, Fang Xu, Ning Xu, \\ Zhiyong Wang \\ Computer Institute of Wuhan University \\ Wuhan, Hubei province, China \\ wang_jing28@126.com; *zhy2536@whu.edu.cn
}

\begin{abstract}
Because the phases of policy management in Mobile ad hoc network (MANET) and the procedure of context awareness in cognitive wireless network are very unclear and complicated. We aim to provide a useful description way to analyze the mechanism of policy-based network management (PBNM) in MANET, the structure of cross-layer components of a context-aware distributed PBM system and the context awareness procedure distinctly. In this paper, we propose a novel Colored Time Petri Net (CTPN) Model to describe the procedure of policy management and context awareness. According to the definition of CTPN and the self-management policies of MANET, the procedure of policy management is divided into 3 phases, and a corresponding CTPN model is designed. Based on the 4 phases of distributed context-aware model, a whole CTPN model of context awareness procedure is presented. And a simplified support graph of context awareness procedure is proposed in order to illustrate the effectiveness of the method.
\end{abstract}

Keywords-Colored Time Petri Net; Mobile ad hoc network; Policy-based network management; Context awareness; Support graph

\section{INTRODUCTION}

Petri net was developed by Petri in 1962 from Carl A dam Petri's doctor dissertation[1]. Nowadays, applications of Petri net are used widely in many fields. And modeling of work flow is an important strongpoint in Petri net because of its analysis abilities in mathematics and graphics. The modeling description languages in Petri net have three forms, such as Timed Petri nets, colored Petri nets, and stochastic Petri nets [2].

Colored Time Petri Net (CTPN) is a available modeling tool in the field of designing, specification, simulation, validation, and implementation of complicated systems and discrete applications [3]. In Dotoli and Fanti [4], CPTN is described detailedly. And CTPN is defined as a bipartite directed graph represented by a $C P T N=\left(\sum, P, T, F, C, I_{-}, I_{+}, \Delta t, M_{0}\right)$ and it satisfies the following requirements:

(1) $\sum$ is called color set, and it is a finite set of nonempty types.

\author{
Xuejun Zhou, Chen Li, Zhao Shen \\ Department of Information and Network Technology, \\ Electronic Engineering School, Naval University of \\ Engineering \\ Wuhan, Hubei province, China \\ 15994249224@139.com
}

(2) $(P, T ; F)$ is base network which is a directed net. Places are represented by $P=\left(p_{1}, p_{2}, \ldots, p_{m}\right)$ which is a finite set, and Transitions are represented by

$$
T=\left(t_{1}, t_{2}, \ldots, t_{n}\right), P \bigcap T=\Phi, P \bigcup T=\Phi .
$$

(3) $C$ is represented by a colored function which is from $P$ into $\sum$. Every token existing in its corresponding $P$ is a resource such as wireless route, which has a value. After a transition, the tokens are consumed.

(4) $I_{-}$and $I_{+}$are defined as a negative and a positive function from $P \times T$, i.e., colored directed arcs from $\mathrm{p}$ to $\mathrm{t}$ and from t to $\mathrm{p}$. For all $(p, t) \in P \times T$ such that:

$$
\begin{aligned}
& \forall p \in P, \exists t \in T: I_{-}(p, t) \neq 0 \cup I_{+}(p, t) \neq 0 \\
& \forall t \in T, \exists p \in P: I_{-}(t, p) \neq 0 \cup I_{+}(t, p) \neq 0
\end{aligned}
$$

(5) $\Delta t: T \rightarrow$ Range, where Range is a firing duration interval of $\mathrm{T}, \quad \operatorname{Range}(T)=\left(a_{\min }, a_{\max }\right) \quad$ and $0 \leq a_{\min } \leq a_{\max }$. For each transition $t \in T$, there is a time interval.

(6) $M_{0}$ is called the function of initialization. $M_{0}$ is defined from $\mathrm{P}$ into closed expressions assigning a color to each place such that:

$$
\forall p \in P: \operatorname{Type}(I(p))=C(p)_{M S} \text {. }
$$

Timed Petri net is classified by two kinds: timed place $\mathrm{P}$ or timed transition T. And there are two ways of transitions. The first is a Timed Transition, from the input to output there is a delay in which a token can be disposed; the second is an Immediate Transition, there is not delay from the input to output, and a token can be transmitted immediately.

A simple process of context awareness is modeled by CTPN in Figure 1. The token in place p0 represents original context information in MANET; place p1, p2 and p3 represent some wireless nodes which possess some special functions; t0, t1 and t2 represent some distribution transitions. After 1-4 minutes, the continuous time of distribution process, through the timed transition t0, token 
in place $\mathrm{p} 0$ is lost and place $\mathrm{p} 1$ receives a token. If the condition is c1, after 3-8 minutes, through the timed transition $\mathrm{t} 1$, place $\mathrm{p} 2$ achieve the token, and if the condition is c2, place p3 get the token immediately through the immediate transition $\mathrm{t} 2$.

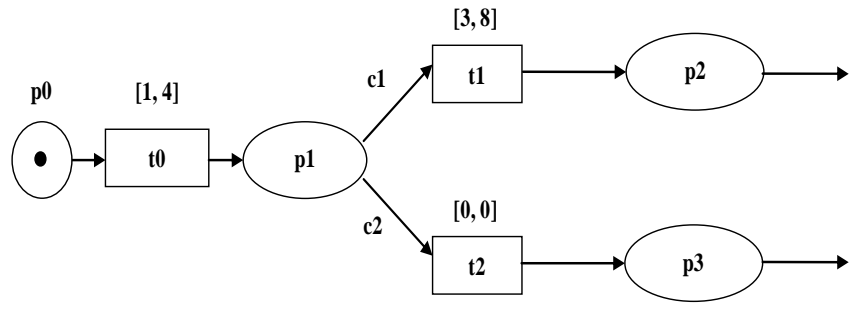

Figure 1. Example of simple model of context awareness procedure based on CTPN in MANET

\section{RELATED WORK}

Recently the related literature in MANET is limited and proposed solutions attempt to only partly solve some traditional issues in order to improve the QoS performance. There were a number of groups working on policies for network and distributed systems management [5,6]. The policy-based paradigm $[7,8]$ offered a promising solution since it allows dynamic alteration and controlled programmability of management logic based on the supported policy types. The method of tackling MANET management was presented in [9]. The suggested Ad hoc network management protocol (ANMP) was based on hierarchical clustering of nodes in a three level architecture. The "Guerilla" architecture [10] adopted an agent-based two-tier distributed approach where at the higher level "nomadic managers" made decisions and launched active probes to fulfill management objectives. The exploitation of context in network management had been addressed. Relevant adaptive systems were deployed, interacting with the surrounding environment and functioning according to relevant conditions [11-14]. The main drawback of all these approaches is the static evaluation of context against predefined rules. In [15,16], Antonis M. Hadjiantonis introduced context information into MANET in order to trigger cross-layer changes according to policies, leading to a degree of autonomic decision-making. And the proposed context-aware PBNM framework which introduces a novel policy-based organizational model is the first to consider exploiting context information which can effectively manage a MANET in conjunction with a PBNM system. Because the effectiveness of $\mathrm{Pi}$-calculus has been proved in the field of web service composition and analysis, and a formal model of service network including the definition of abstract service and semantic structure of channel was described by Pi-calculus in [17].

In this paper, we propose a novel CTPN model for the procedure of context awareness and policy management in MANET, and a simplified support graph of context awareness procedure can illustrate the effectiveness of the method.

\section{Procedure of Policy Management AND CONTEXT AWARENESS IN MANET}

Mobile ad hoc network (MANET) is an emerging paradigm in wireless networks. MANET has many inherent advantages, such as unrestrained computing, lacking centralization and ease of deployment at low costs.

In MANET, mobile nodes move randomly and are organized by themselves arbitrarily; therefore, the topology structure of network changes unpredictably and rapidly. And the fixed infrastructure of network and centralized deployment exist in conventional wireless network. But it is different, MANET is self-creating, every node try its best to discover other nodes which it can communicate with dynamically. The pattern of its dynamic self-creation often requires an ability of self-management according to higherlevel organization policies, such as the context of the surrounding conditions.

\section{A. Self-management Policies}

Through the utility of some policies of management, the some QoS performances will be promoted. Especially the problem of throughput and failure of cooperation with other WLANs exist in MANET, so the performance will be lower and can not satisfy the requirements of users. There exists the interference between different MANETs which are based on the base station mostly. From the above discussion, it is not difficult for us to find that if the devices can randomly choose the channel, and it exists a negative influence on the performance of MANETs. For this reason, the selfmanagement policies of MANET were designed from [18].

From the table 1, we can find that there are 8 policies in order to allow the devices access MANET dynamically and choose the best channel by making use of the information of the MAC layer of every devices.

According to policies, context information can be used to trigger cross-layer changes. In Figure 2, a context-aware PBM system is presented.

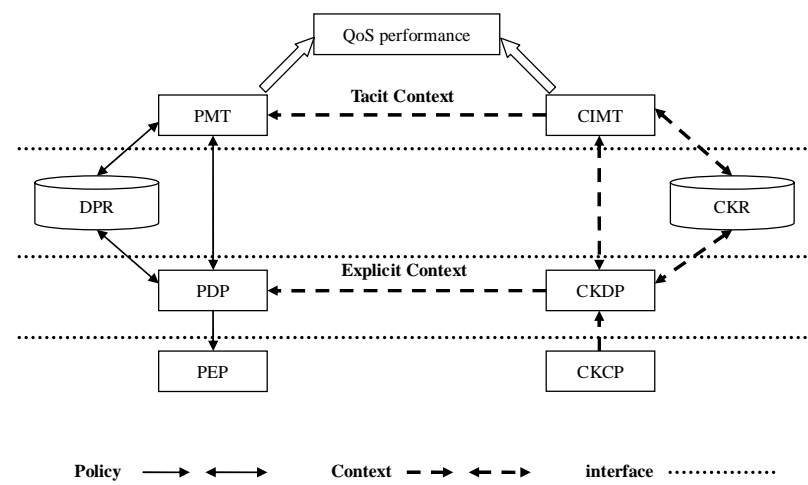

Figure 2. Cross-layer components of a context-aware distributed PBM system 
TABLE II SELF-MANAGEMENT POLICIES OF MANET

\begin{tabular}{|c|c|}
\hline No. & if $\{$ Conditions $\}$ then $\{$ Actions $\}$ \\
\hline P1 & $\begin{array}{l}\text { if }\{\text { ready }\} \text { then }\{\text { channelsScan()\}, } \\
\text { \{generateCompleteScan(results) }\}\end{array}$ \\
\hline $\mathrm{P} 2$ & $\begin{array}{c}\text { if }\{\text { otherDetectedWLAN=true }\} \text { and } \\
\{\text { FC:=freeChannels(results), FC=true }\} \text { and } \\
\{\text { PC:=preffered(FC,ch_list), PC=true }\} \\
\text { then }\{\text { optimizeChannel(PC, } \\
\left.\left.\text { algorithm }\left(\text { criteria }_{1}\right)\right)\right\}\end{array}$ \\
\hline P3 & 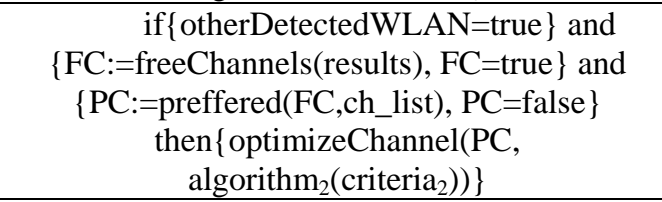 \\
\hline P4 & $\begin{array}{c}\text { if }\{\text { otherDetectedWLAN=true }\} \text { and } \\
\{\mathrm{FC}:=\text { freeChannels(results), FC=false }\} \\
\text { then }\{\text { optimizeChannel(all, } \\
\left.\left.\text { algorithm }{ }_{3}\left(\text { criteria }_{3}\right)\right)\right\}\end{array}$ \\
\hline P5 & $\begin{array}{l}\text { if }\{\text { dynamicallyAdapt }=\text { true }\} \text { then } \\
\text { \{generateStartAdapt(newWLANInfo) }\end{array}$ \\
\hline P6 & $\begin{array}{c}\text { if }\left\{\text { LinkDelay }<\text { threshold }_{\mathrm{a}}\right\} \text { and } \\
\text { \{dynamicallyAdapt }=\text { true }\} \text { then } \\
\{\text { generateAdaptStart(catchedWLANInfo) }\}\end{array}$ \\
\hline P7 & $\begin{array}{c}\text { if }\{\text { channel_distance }(\text { WLANInfo,current })< \\
\text { distance }\} \text { and }\left\{\text { Metric }<\text { threshold }_{\mathrm{b}}\right) \\
\text { then }\{\text { scanChannels }()\},\left\{\text { generateNewAdaptChan }_{\text {nel(results })\}}\right.\end{array}$ \\
\hline P8 & 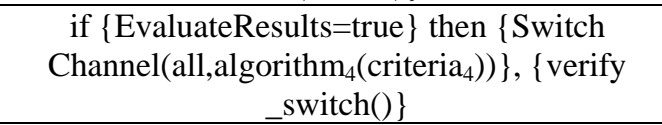 \\
\hline
\end{tabular}

Being different with the distributed PBM system, context-aware model compose 4 elements, Context Intelligence Management Tool (CIMT), Context Knowledge Repository (CKR), Context Decision-making Point (CKDP) and Context Policy Enforcement Point (CKCP). The flow of context information is reverse to the PBM system. Context is collected and processed in lower layers, and policy decisions are made in the higher layers.

The CKCP is established on every wireless node which is in charge of monitoring the status of nodes and environment information by using the professional sensors. And this context information of CKCP is pretreated and transported to its respective CKDP which aggregates the explicit context knowledge to get its special interest and requirement. The aggregated context information is sent to CKR, PDP and CIMT. After reasoning, CIMT gain the tacit context information and send it to PMT, and according to the tacit context knowledge, CKR can learn the tacit interest and character.

Because context awareness can dynamically adapt to the conditions of changing, context information can be transmitted from lower layers to upper layers in order to change the configurations of network and application.

\section{CTPN MOdEL OF POLICY MANAGEMENT AND CONTEXT AWARENESS IN MANET}

According to the description of self-management policies of MANET in III, the CTPN model of policy management procedure is presented in Figure 3. It consists of two main phases corresponding to eight policies shown in Table 1. In the first phase, ad hoc network is initializing through four policies. And in the second phase, the deployed ad hoc network is completing the procedure of dynamical adaptation by four policies.

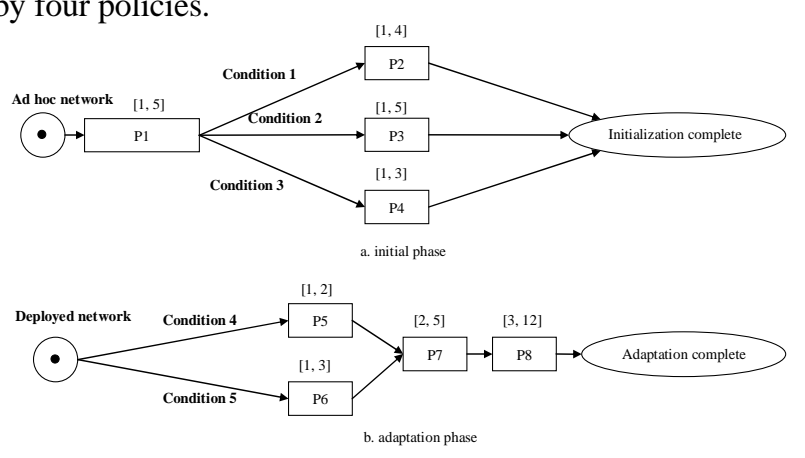

Figure 3. CTPN model of policy management procedure

Through studying the basic components of a policybased management system and the cross-layer components of a context-aware distributed PBM system, we can design 4 CTPN sub-models of context awareness procedure in MANET:

\section{A. Phase of aggregation and pretreatment}

Each wireless node of ad hoc networks is equipped with the corresponding CKCP, which gets the raw context information, including the status of nodes and information of environment. At the same time, simple preprocessing is performed by CKCP, which building the basis for the posterior reasoning and use. In the figure 4, 1 and 3 in the bracket represent the earliest and latest trigger time for the timed transition in which the length of interval is t0.

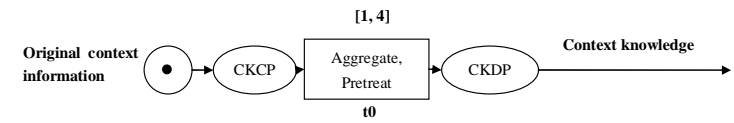

Figure 4. CTPN model of aggregation and pretreatment phase

\section{B. Phase of reasoning and planning}

The context information needs to be reasoned further in order to get the implied useful knowledge through the timed transition 0 in the figure 5 . In the reasoning procedure, the timed transition t0 is classified into 2 conditions: emergent event and common event. If it is the former, the priority of context information is very high, or the period of time of making decision and policy execution is very short, and in the condition of emergent event, the implied useful context information is sent directly to PDP. If it is the latter, the context information is sent to PMT, the feedback result of 
policy implementation is assessed by PMT, and the plan is made for the next step through the timed transition $\mathrm{t} 1$.

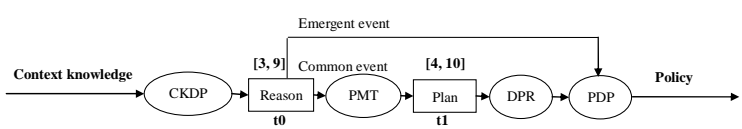

Figure 5. CTPN model of reasoning and planning phase

\section{Phase of learning}

After reasoning the tacit context, the knowledge is analyzed and studied by CIMT, and useful context knowledge is saved into CKR through the timed transition t0, then is sent to DPR. And if the context knowledge is explicit, it is sent directly to CKR by CKDP through the immediate transition $\mathrm{t} 1$, and is saved into the context knowledge repository in the figure 6 .

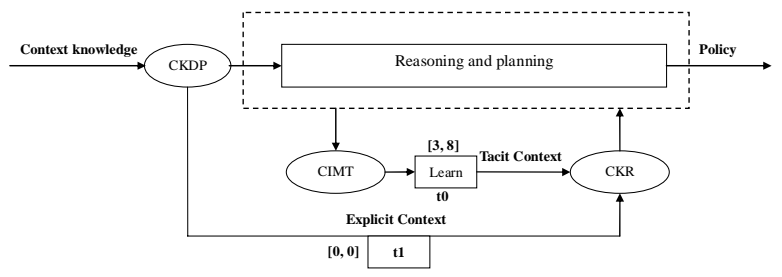

Figure 6. CTPN model of learning phase

\section{Phase of decision-making}

All appropriate policies are decision-made by PDP through the timed transition $\mathrm{t} 0$ and sent to its corresponding PEPs which execute the policies through the timed transition $\mathrm{t} 1$ in the figure 7 . And in result the performance of QoS increases.

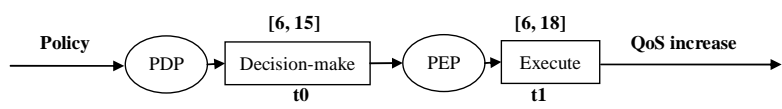

Figure 7. CTPN model of decision-making phase

So the whole CTPN model of context awareness procedure in MANET can be constructed by 4 phases in the figure 8.

Because CTPN model has a capability of description, the procedure of context awareness in the figure 8 is simplified into a support graph in the figure 9. Input condition is a decrease of the QoS performance of MANET, and through events E1(Aggregation and pretreatment), E2(Reasoning and planning), E3(Learning), E4(Decision-making), the output result can be achieved which is an increase of the QoS performance.

\section{CONCLUSIONS}

CTPN is an effective and visual tool with an abstraction mechanism which is always utilized in system simulation and analysis. This paper presents a novel model of context awareness procedure and policy management in MANET using CTPN. According to some policies of selfmanagement in MANET and cross-layer components of a context-aware distributed PBM system, the whole model of policy management procedure and Context Awareness procedure in MANET are presented in this paper by the CTPN tools. And a simplified support graph of context awareness procedure can illustrate the effectiveness of the method.

\section{ACKNOWLEDGMENT}

Wang Jing thanks for being supported by the National Natural Science Foundation of China under Grant No. 61272454 and the Specialized Research Fund for the Doctoral Program of Higher Education of China under Grant No. 20130141110022.

\section{REFERENCES}

[1] PETRI C. A. Kommunikation Wit Automaten. Bohn: University of Bohn, 1962, pp. 14-16.

[2] Zang Daxin, Shi Xiaoding, Jin Maozhong. Modeling and scheduling of real-life assembly job shop with timed colored Petri net. 2010 7th international conference on Service Systems and Service Management, 2010. pp. 484-488.

[3] Huang, S., Hu, Y. J., Li, C. G. A TCPN based approach to model the coordination in virtual manufacturing organizations. Computer \& Industrial Engineering, 2004. pp. 61-76.

[4] Dotoli, M., Fanti, M. P. An urban traffic network model via coloured timed Petri nets. Control Engineering Practice, 2006. pp. 1213-1229.

[5] Koch, T. et al. Policy Definition Language for Automated Management of Distributed System. 2nd IEEE Int. Workshop on Systems Management, Toronto, June 1996, pp. 55-64.

[6] Wies R. Policies in Integrated Network and Systems Management: Methodologies for the Definition, Transformation and Application of Management Policies. Ph.D. Dissertation, Fakultat fur Mathematik der Ludwig-Maximilians-Universitat, Munchen, Germany, 1995, pp. 25-28.

[7] Ritu Chadha, Yuu-Heng Cheng, Jason Chiang, et al. Policy Based Mobile Ad hoc Network Management. 5th IEEE International Workshop on Policies for Distributed Systems and Networks (POLICY2004), June 2004, pp. 112-119.

[8] Phanse, K.S., DaSilva, L.A. Protocol support for policy-based management of mobile ad hoc networks. IEEE/IFIP Network Operations and Management Symposium (NOMS), 2004, pp. 75-81.

[9] Chen W., Jain N., Singh, S. ANMP Ad hoc network management protocol. IEEE Journal on Selected Areas in Communications, Vol 17(8), Aug.1999, pp. 88-94.

[10] Shen C., Srisathapornphat C., Jaikaeo C. An adaptive management architecture for ad hoc networks. IEEE Communication Magazine, Vol 41(2), Feb.2003, pp. 151-158.

[11] A. Malatras, G. Pavlou. Context-driven Self-Configuration of Mobile Ad hoc Networks. IFIP International Workshop on Autonomic Communications (WAC 2005), October 2005, pp. 95-103.

[12] Bellavista, P., Corradi, A., Montanari, R., et al. Context-aware middleware for resource management in the wireless Internet. IEEE Transactions on Software Engineering, Vol 29(12), December 2003, pp. 133-139.

[13] Yau, S.S., Karim, F., Wang, Y., et al. Reconfigurable ContextSensitive Middleware for Pervasive Computing. IEEE Pervasive Computing, July-September 2002, pp. 75-82.

[14] S. Gouveris, S. Sivavakeesar, G. Pavlou, et al. Programmable Middleware for the Dynamic Deployment of Services and Protocols 
in Ad Hoc Networks. IEEE/IFIP Integrated Management Symposium (IM 2005), May 2005, pp. 148-154.

[15] Antonis M. Hadjiantonis, Apostolos Malatras, et al. A context-aware, policy-based framework for the management of MANETs. Proceedings of the Seventh IEEE International Workshop on Policies for Distributed Systems and Networks (POLICY'06), 2006, pp. 199206.

[16] Apostolos Malatras, Antonios M. Hadjiantonis, and George Pavlou. Exploiting Context-Awareness for the Autonomic Management of Mobile Ad Hoc Networks. Journal of Network and Systems Management, Vol. 15, No. 1, March 2007. pp. 29-55.
[17] Hu Jing, Feng Zhi-Yong. A formal model of service network and its description using pi-calculus. Proceedings of 2012 International Conference on Computer Science and Service System, CSSS 2012. pp. 1476-1479.

[18] Lu Chuntao, Zhang Huyin, Wu Di. The Research on Policymanagement-based Dynamic Spectrum Access Technology for Cognitive Network. 2009 ISECS International Colloquium on Computing, Communication, Control, and Management, 2009. pp. 184-187.

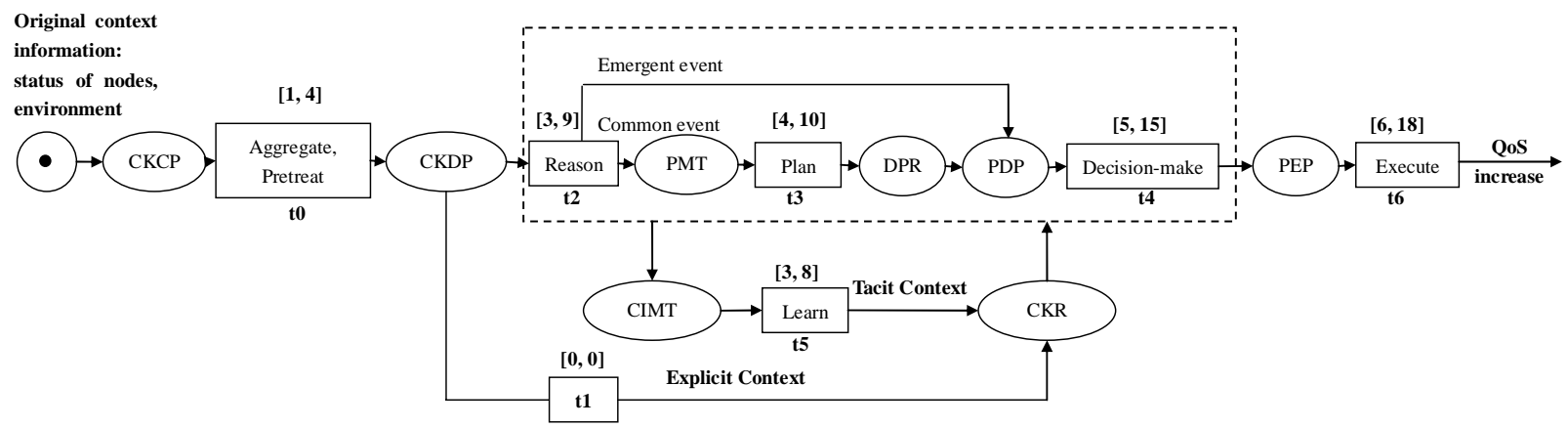

Figure 8. Whole CTPN model of context awareness procedure in MANET

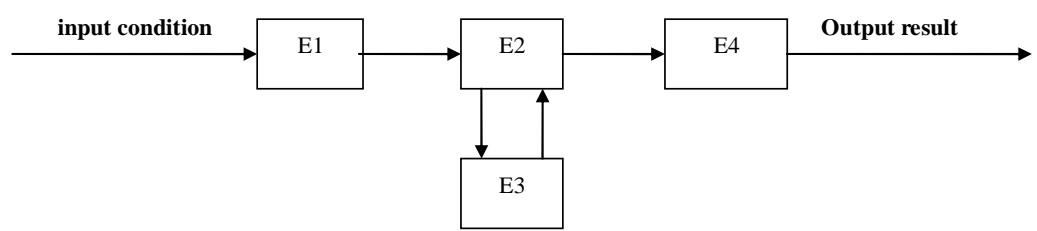

Figure 9. Simplified support graph of context awareness procedure 